\title{
Antidepressant side effects and their impact on treatment outcome in people with major depressive disorder: an iSPOT-D report
}

\author{
Taylor A. Braund (D) ${ }^{1,2}$, Gabriel Tillman ${ }^{3}$, Donna M. Palmer ${ }^{1,4}$, Evian Gordon ${ }^{5}$, A. John Rush ${ }^{6,7,8}$ and Anthony W. F. Harris (iD ${ }^{1,2}$
}

(c) The Author(s) 2021

Side effects to antidepressant medications are common and can impact the prognosis of successful treatment outcome in people with major depressive disorder (MDD). However, few studies have investigated the severity of side effects over the course of treatment and their association with treatment outcome. Here we assessed the severity of side effects and the impact of treatment type and anxiety symptoms over the course of treatment, as well as whether side effects were associated with treatment outcome. Participants were $N=1008$ adults with a current diagnosis of single-episode or recurrent, nonpsychotic MDD. Participants were randomised to receive escitalopram, sertraline, or venlafaxine-extended release with equal probability and reassessed at 8 weeks regarding Hamilton Rating Scale Depression $\left(\mathrm{HRSD}_{17}\right)$ and Quick Inventory of Depressive Symptomatology (QIDS-SR $\left.{ }_{16}\right)$ remission and response. Severity of side effects were assessed using the Frequency, Intensity, and Burden of Side Effects Rating (FIBSER) scale and assessed at day 4 and weeks 2, 4, 6, and 8. Frequency, intensity, and burden of side effects were greatest at week 2, then only frequency and intensity of side effects gradually decreased up to week 6 . Treatment type and anxiety symptoms did not impact the severity of side effects. A greater burden_but not frequency or intensity-of side effects was associated with poorer treatment outcome and as early as 4 days post-treatment. Together, this work provides an informative mapping of the progression of side effects throughout the treatment course and their association with treatment outcome. Importantly, the burden of side effects that are present as early as 4 days post-treatment predicts poorer treatment outcome and should be monitored closely. iSPOT-D: Registry name: ClinicalTrials.gov. Registration number: NCT00693849.

Translational Psychiatry (2021)11:417 ; https://doi.org/10.1038/s41398-021-01533-1

\section{INTRODUCTION}

Selective serotonin reuptake inhibitors (SSRIs) and serotonin-norepinephrine reuptake inhibitors (SNRIs) are firstline pharmacological treatments for people with major depressive disorder (MDD) [1] and anxiety disorders [2]. However, SSRIs and SNRIs are associated with a range of side effects, including loss of appetite, weight loss, drowsiness, dizziness, fatigue, headaches, increased suicidal thoughts, nausea/vomiting, sexual dysfunction, and increased risk of cardiovascular and cerebrovascular events [3-5]. While specific antidepressant side effects are diverse, they also occur generally with a high frequency, intensity, and burden $[6,7]$. Previous studies suggest that frequency, intensity, and burden of side effects are impacted by antidepressant treatment type, anxiety symptoms, and the presence of anxious depression (for a review, see lonescu et al. [8]).

Anxious depression is present in approximately half of the MDD population and is commonly defined either syndromally (i.e., cooccurring MDD and anxiety disorder diagnoses) or using 17-item Hamilton Rating Scale Depression $\left(\mathrm{HRSD}_{17}\right)$ criteria (i.e., MDD diagnosis and an $\mathrm{HRSD}_{17}$ anxiety/somatisation factor score of $\geq 7$
[9-11]). Some, but not all studies suggest that anxious depression increases the severity of side effects (for a review, see lonescu et al. [8]). For example, people with anxious depression were found to have a greater maximum frequency, intensity, and burden of side effects compared to people with non-anxious depression in the Sequenced Treatment Alternatives to Relieve Depression (STAR*D) trial [12]. A greater side effect severity has also been found at various study endpoints in people with anxious depression compared to people with MDD only [13-15]. However, these associations are not found in all definitions of anxious depression, with Gaspersz et al. [14]. finding no differences in the frequency of side effects between people with syndromal and non-syndromal anxious depression.

Few studies have assessed how side effects progress throughout treatment and their association with treatment outcome [8]. Given that the presence of anxiety symptoms and treatment type may impact side effect profiles [8], the aim of the current study was to assess the severity of side effects - as well as the impact of anxiety symptoms and treatment type-over the course of treatment in a large patient sample from the international Study to Predict

\footnotetext{
${ }^{1}$ Brain Dynamics Centre, The Westmead Institute for Medical Research, Sydney, NSW, Australia. ${ }^{2}$ Discipline of Psychiatry, Sydney Medical School, University of Sydney, Sydney, NSW, Australia. ${ }^{3}$ School of Science, Psychology and Sport, Federation University, Ballarat, VIC, Australia. ${ }^{4}$ Total Brain, Sydney, NSW, Australia. ${ }^{5}$ Total Brain, San Francisco, CA, USA. ${ }^{6}$ Department of Psychiatry \& Behavioral Sciences, Duke University, School of Medicine, Durham, NC, USA. ${ }^{7}$ Department of Psychiatry, Texas Tech University Health Sciences Center, Lubbock, TX, USA. ${ }^{8}$ Professor Emeritus, Duke-National University of Singapore, Singapore, Singapore. ${ }^{凶}$ email: taylor.braund@sydney.edu.au
} 
Optimized Treatment for Depression (iSPOT-D) [16]. We also tested whether early ratings of side effects predicted treatment outcome. We expected that, in line with previous studies [8], treatment type and anxiety symptoms would impact the frequency, intensity, and burden of side effects. We also expected that higher side effect ratings would predict poorer treatment outcome.

\section{METHODS \\ Study overview}

The iSPOT-D is a phase-IV, multi-site, international, randomized, open-label trial designed to identify markers of treatment response to commonly prescribed medications in an adult depressed, outpatient population. All participants were either antidepressant medication naive or washed out. Participants were randomized to receive escitalopram, sertraline, or venlafaxine-extended release (venlafaxine-XR) with equal probability. Assessments were collected at pre-treatment and post-treatment at 8 weeks. Study site personnel contacted participants by telephone at day 4 and weeks 2, 4, and 6 to monitor antidepressant dosage, compliance, concomitant medications, and adverse events. The iSPOT-D trial was designed with no placebo arm and participants were aware of the medication that they were taking to best match real-world practice. In this way, findings also reflect treatment regimens that exist in routine practice and promote the translatability of the findings. Data were collected between December 1, 2008 and September 30, 2013. For more details on the study protocol design, rationale and methods, see Williams et al. [16].

\section{Participants}

Participants $(N=1008)$ were adults (age $18-65$ years) with a current diagnosis of single-episode or recurrent, nonpsychotic MDD (CONSORT chart provided in Supplementary Fig. 1). Participants were diagnosed on the Mini-International Neuropsychiatric Interview_Plus (MINI-Plus) [17] according to Diagnostic and Statistical Manual of Mental Disorders (DSM)IV criteria [18]. All participants required a $\mathrm{HRSD}_{17}$ score $>16$ at entry. For a full list of inclusion and exclusion criteria, see Supplementary Fig. 2. Participants provided written informed consent after receiving a complete description of the study. The study was approved by institutional or ethical review boards at each site, and its protocols followed International Conference on Harmonization and Good Clinical Practice principles, the U. S. Food and Drug Administration Code of Federal Regulations, and country-specific guidelines (see Supplementary Table 1 for individual iSPOT-D study management sites and investigators).

\section{Depression severity}

Depression severity was assessed using the $\mathrm{HRSD}_{17}$ [19] and the 16-Item Quick Inventory of Depressive Symptomatology-Self-Rated (QIDS-SR 16 ) $[20,21]$. The $\mathrm{HRSD}_{17}$ is a 17-item clinician-rated scale scored either on a 3point or 5-point Likert scale used to rate severity of their depression by gauging mood, feelings of guilt, suicide ideation, insomnia, agitation or retardation, anxiety, weight loss, and somatic symptoms [19]. The QIDS$\mathrm{SR}_{16}$ is a 16-item questionnaire scored on a 4-point Likert scale used to rate the severity of depression by assessing the severity of the nine DSM-IV diagnostic symptom domains for MDD. Remission was defined as a week 8 HRSD $_{17}$ score $\leq 7$ or a week 8 QIDS-SR 16 score $\leq 5[20,21]$. Response was defined as a $\geq 50 \%$ decrease from baseline on the HRSD 17 or QIDS-SR 16 . In line with the study protocol, the primary outcomes were rates of remission and response on the HRSD ${ }_{17}$. The secondary outcome was remission and response on the QIDS-SR 16 . The $\mathrm{HRSD}_{17}$ has shown good internal consistency $(a=79)$ and test-retest reliability $(r=0.87)$ in a recent metaanalysis [22]. The QIDS-SR ${ }_{16}$ has also shown good internal consistency $(a=$ $0.86)$, as well as convergent and discriminant validity $[20,23]$.

\section{Frequency, intensity, and burden of side effects}

Side effect severity was measured using the self-reported Frequency, Intensity, and Burden of Side Effects Rating (FIBSER) [24] scale. The FIBSER assesses three domains of antidepressant medication side effect impact, including Frequency (frequency of side effects of medications taken within the past week for depression), Intensity (intensity of side effects due to medications taken within the last week for depression), and Burden (degree to which antidepressant medication side effects over the last week interfered with day-to-day functions). Frequency, Intensity, and Burden was rated on a 7-point scale, ranging from "no side effects" to "present all the time" for frequency, from "no side effects" to "intolerable" for intensity, and from "no impairment" to "unable to function due to side effects" for burden (see Supplementary Table 2 for categorical distribution of FIBSER scores at each study timepoint).

\section{Adverse events}

Adverse events were recorded and coded using the Medical Dictionary for Regulatory Activities (MedDRA) [25]. MedDRA is a multi-axial, five-tiered hierarchical terminology used by regulatory authorities and the biopharmaceutical industry for the coding and classification of adverse events. Adverse events were reported using their highest hierarchical grouping (i.e., system organ classes). Adverse events were determined as related to the study treatments (i.e., potential side effect to study treatment) by the investigator and in line with the study protocol. The terminology was developed and is endorsed by the International Conference on Harmonization of Technical Requirements for Registration of Pharmaceuticals for Human Use.

\section{Anxious depression definitions}

Syndromal anxious depression. Syndromal anxious depression was defined as a DSM-IV MDD diagnosis and at least one concurrent MINIPlus identified anxiety disorder, including generalized anxiety disorder, panic disorder, agoraphobia, social phobia, and specific phobia [9].

$H R S D_{17}$ anxious depression. HRSD 17 anxious depression was defined as a DSM-IV MDD diagnosis and a HRSD 17 anxiety/somatization factor score of $\geq 7$ [9]. The anxiety/somatisation factor was derived from a factor analyses of the HRSD 17 by Cleary and Guy [26] and includes six items: hypochondriasis, insight, general and gastrointestinal somatic symptoms, and psychic and somatic anxiety.

\section{Depression, anxiety, and stress scale}

Anxiety was also assessed using the self-rated 42-item Depression Anxiety and Stress Scale $\left(\mathrm{DASS}_{42}\right)[27,28]$. DASS 42 item scores range from 0 (did not apply to me at all) to 3 (applied to me very much or most of the time). The 14-item DASS ${ }_{42}$ anxiety subscale, used in this study, includes 14 items measuring autonomic arousal, skeletal muscle effects, situational anxiety, and subjective experience of anxious affect.

\section{Protocol treatment}

Participants were randomized to receive escitalopram, sertraline, or venlafaxine-XR with equal probability. All psychotropic medications (except sleep aids and anxiolytics) were discontinued and washed out prior to baseline assessments. Antidepressants were prescribed and doses were adjusted by the participant's treating physician according to routine clinical practice. Additional medication for associated symptoms (e.g., insomnia) or medication-induced side effects (e.g., nausea) were allowed as they reflect common practice. Any treatment for concurrent general medical conditions, except medications contraindicated with the studyassigned antidepressants, were allowed and recorded.

\section{Statistical analysis}

To test whether timepoint, treatment type (i.e., individual treatment arm), DASS $_{42}$ anxiety subscale, $\mathrm{HRSD}_{17}$ anxiety/somatization, and presence of anxious depression were associated with side effects, we used hierarchical linear mixed effect models. Mixed effect models are well suited to the data as they retain participants with missing data and allow for the post hoc tests. We used a model comparison approach with the Akaike Information Criterion (AIC) [29] and Bayesian Information Criterion (BIC) [30], which selects the best fitting model while penalizing for complexity (i.e., number of parameters in the model). All models included side effect scores as the dependent variable, included subjects as a random effect, and included the fixed effect covariates of age, sex, baseline depression severity (measured via the QIDS-SR ${ }_{16}$ ), dosage at study outcome, and age of MDD onset. We then tested a set of models that either added the fixed effect variable timepoint or the fixed effect variables timepoint + treatment type, timepoint $+\mathrm{DASS}_{42}$ anxiety, timepoint $+\mathrm{HRSD}_{17}$ anxiety/somatization, or timepoint + anxious depression. Continuous fixed effects of age, depression severity, $\mathrm{DASS}_{42}$ anxiety, $\mathrm{HRSD}_{17}$ anxiety/somatization, age of MDD onset, and treatment dosage were grand mean centered. Post hoc $t$ tests were performed using estimated marginal means and corrected for using the Bonferroni-Holm [31] method. 
Pearson correlations were used to test for associations between severity of side effects and the QIDS-SR ${ }_{16}$ total score at each study timepoint. Logistic regression models were used to test whether the severity of side effects at each study timepoint were associated with antidepressant treatment outcome. Logistic regression models were also adjusted for the same covariates as those used in the hierarchical linear mixed effect models to test whether side effects were associated with treatment outcome independent of these covariates.

All analyses were conducted using R 3.5.1 [32]. Mixed linear models were tested using the "Ime4" package in R [33]. $p$ Values for mixed linear models were calculated using the "ImerTest" package in R [34]. Post hoc tests were performed using the "emmeans" package in $\mathrm{R}$ [35].

\section{RESULTS}

\section{Demographics}

iSPOT-D demographics have been published elsewhere [6]. Briefly, there were 571 females (56.6\%), with a mean age of $37.9(\mathrm{SD}=12.6), 14.5(\mathrm{SD}=2.8)$ years of education, 22.28 $(S D=12.0)$ age of MDD onset, and a QIDS-SR 16 baseline score of $14.5(\mathrm{SD}=3.8)$. Syndromal anxious depression was present in 261/1008 (25.9\%) and $\mathrm{HRSD}_{17}$ anxious depression was present in $422 / 1008$ (41.9\%), with a mean $\mathrm{HRSD}_{17}$ anxiety/ somatisation score of $6.16(S D=1.90$; see Supplementary Fig. 3 for distribution of $\mathrm{HRSD}_{17}$ anxiety/somatisation factor scores). Mean dosages at week 8 were $12.5 \mathrm{mg} /$ day $(S D=9.3)$ for escitalopram, $59.6 \mathrm{mg} /$ day $(\mathrm{SD}=33.2)$ for sertraline, and $76.1 \mathrm{mg} /$ day $(S D=41.5)$ for venlafaxine-XR. Forty-two participants dropped out due to safety, tolerability, or efficacy reasons (see Supplementary Table 3 for distribution of last recorded FIBSER scores).

\section{Frequency, intensity, and burden of side effects}

Table 1 shows the model comparison results for the hierarchical linear mixed effect models. The best fitting models overall (i.e., "the timepoint models") included subjects as a random effect, the fixed effect timepoint, as well as the fixed effect covariates of age, sex baseline depression severity, dosage at study outcome, and age of MDD onset (frequency timepoint model: $A I C=9970.9$, $\mathrm{BIC}=10042.6 ; X^{2}(4)=61.77, p<001$, intensity timepoint model: $\mathrm{AIC}=9393.1, \mathrm{BIC}=9464.8 ; X^{2}(4)=93.99, p<001$, burden timepoint model: $\left.\mathrm{AIC}=8409.0, \mathrm{BIC}=8480.7 ; X^{2}(4)=57.96, p<001\right)$. However, including treatment type, DASS $_{42}$ anxiety, HRSD 17 anxiety/somatization, or the presence of anxious depression did not improve the model's fit to the data (for significant covariates, see Supplementary Material). Results did not change when removing those who dropped out due to safety, tolerability, or efficacy reasons (see Supplementary Table 4).

We also tested whether people with anxious depression had a greater maximum frequency, intensity and burden of side effects compared to people with non-anxious depression. However, there were no differences in the maximum frequency, intensity, or burden of side effects in people with HRSD or syndromal anxious depression compared to non-anxious depression (see Supplementary Table 5).

\section{Post hoc tests}

Figure 1 shows the mean FIBSER score trajectories (for all post hoc test results, see Supplementary Table 6). Post hoc tests revealed that, compared to day 4 , there was a greater frequency, intensity, and burden of side effects at weeks $2,4,6$, and 8 (all $p<0.001)$. Frequency and intensity of side effects at week 2 were greater compared to weeks 6 (both $p=0.002)$ and $8(p=$ 0.012 and 0.009 , respectively), but not week 4 . Frequency and intensity of side effects at week 4 were greater compared to weeks 6 ( $p=0.012$ and 0.006 , respectively), but only intensity was greater compared to week $8(p=0.020)$. There were no differences in frequency, intensity, and burden of side effects between weeks 6 and 8 .

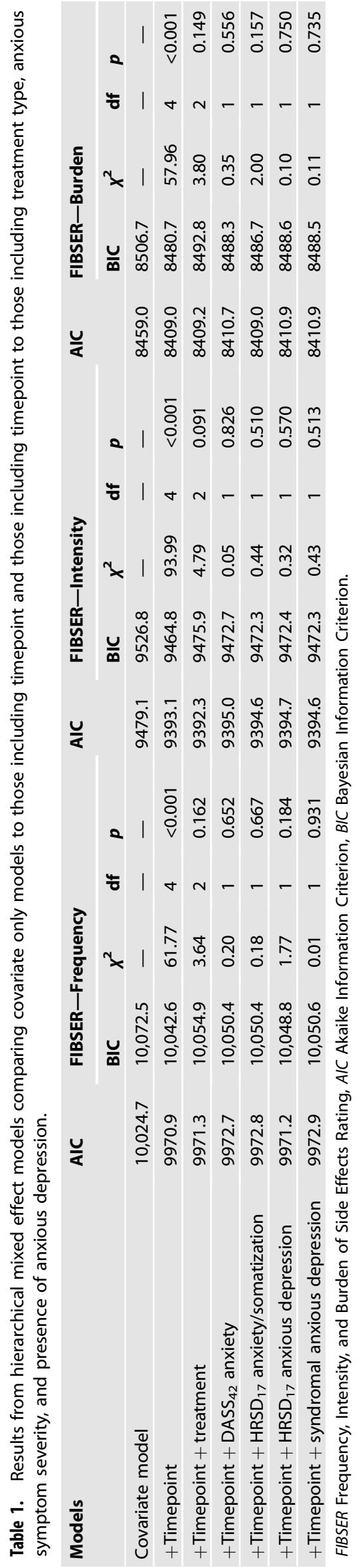




\section{Correlations between QIDS-SR $\mathbf{1}_{\mathbf{1 6}}$ and side effects}

Table 2 shows the correlations between QIDS-SR 16 scores and FIBSER scores at each study timepoint. Only burden of side effects was significantly but weakly correlated with QIDS-SR $_{16}$ scores at day 4. Following day 4, FIBSER scores showed significant weak positive correlations with QIDS-SR ${ }_{16}$ at each study timepoint. The strength of correlations between the FIBSER and QIDS-SR ${ }_{16}$ at each study timepoint increased as the study progressed up to week 6 , then decreased at week 8 .

\section{Side effects predicting antidepressant treatment outcome}

Table 3 shows the results from logistic regression models testing whether frequency, intensity, and burden of side effects predicted $\mathrm{HRSD}_{17}$ and $\mathrm{QIDS} \mathrm{SR}_{16}$ remission and response after adjusting for covariates age, sex, baseline depression severity (measured via the QIDS-SR ${ }_{16}$ ), dosage at study outcome, and age of MDD onset. Figure 2 shows the side effect trajectories of $\mathrm{HRSD}_{17}$ and $\mathrm{QIDS} \mathrm{SR}_{16}$ remitters and responders. A greater burden of side effects at week 2 was associated with poorer $\mathrm{HRSD}_{17}$ remission, and greater intensity of side effects at week 6 was associated with poorer $\mathrm{HRSD}_{17}$ remission. A greater burden of side effects was associated with poorer QIDS-SR ${ }_{16}$ remission and response at every timepoint, except for QIDS-SR ${ }_{16}$ response at week 4. A greater intensity of side effects at week 2 was also associated with poorer remission.

\section{Adverse events}

Four hundred and seventy-three (46.9\%) participants experienced an adverse event between baseline and week 8 . Supplementary Table 7 shows the distribution of adverse events related to different system organ classes. Supplementary Table 8 shows the distribution of individually reported adverse events. One thousand and sixteen adverse events were reported in iSPOT-D. Of these, $804(79.1 \%)$ were reported as being related to the antidepressant

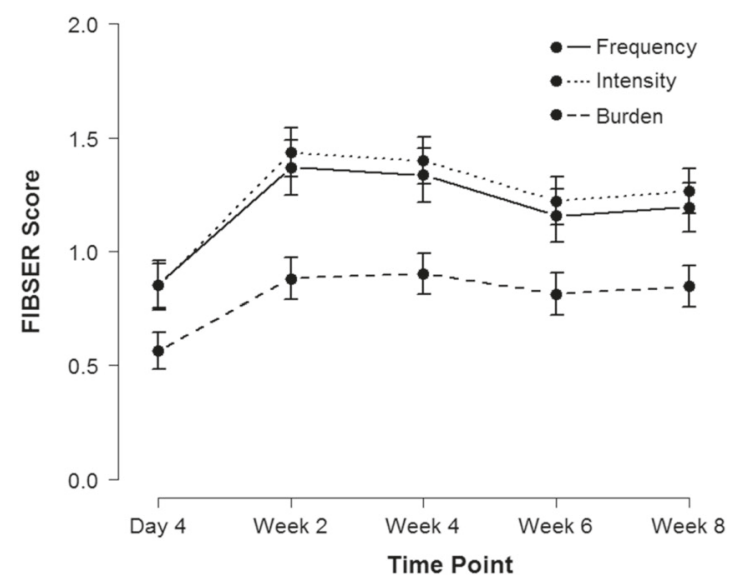

Fig. 1 Mean FIBSER score trajectories. Note: Error bars represent $95 \%$ confidence intervals of the mean. treatments. The most common adverse events reported that were likely related to the study treatments were psychiatric disorders $(28.6 \%, 230 / 804)$, gastrointestinal disorders $(27.6 \%, 222 / 804)$ nervous system disorders $(16.3 \%, 131 / 804)$, general disorders $(12.1 \%, 97 / 804)$, and metabolism and nutrition disorders (5.7\%, 46/ 804). However, the presence of these adverse events were not associated with $\mathrm{HRSD}_{17}$ or $\mathrm{QIDS}^{-\mathrm{SR}_{16}}$ remission and response (all $p>0.05)$.

\section{DISCUSSION}

These findings are the first to comprehensively outline the progression of side effect severity throughout the course of antidepressant treatment and their impact on treatment outcome. We found side effects were greatest at week 2, then gradually decreased up to week 6 . Treatment type and anxiety symptoms did not impact the severity of side effects over the course of treatment. Finally, burden of side effects-but not frequency or intensity of side effects-were associated with poorer treatment outcome and as early as 4 days posttreatment.

Frequency, intensity, and burden of side effects increased from day 4 to week 2 post treatment. However, only frequency and intensity of side effects decreased after 4 weeks of treatment. Identifying the point at which side effects begins to recede has important clinical implications, as it may take up to 6 weeks for patients to begin seeing a reduction in side effect frequency and intensity. However, burden of side effects did not decrease after 4 weeks of treatment and endured throughout the course of antidepressant treatment. The enduring perceived burden of side effects is a commonly reported issue that contributes to the myriad of issues related to treatment adherence and efficacy [36]. Furthermore, depression severity was only weakly correlated with side effects, suggesting that the reduction of frequency and intensity of side effects was not just a proxy of a reduction in depression severity. Taken together, burden of side effects fail to decrease over the course of treatment and should be considered when assessing treatment suitability for patients in clinical practice.

Previous studies suggest that treatment type and anxiety symptoms may impact the severity of antidepressant side effects $[8,12]$. However, treatment type, anxiety symptom severity, and the presence of anxious depression did not impact the severity of side effects in the current study. For example, previous studies have found a greater maximum frequency, intensity, and burden of side effects compared to people with non-anxious depression [12] and a greater side effect severity at study endpoints [13]. Here we investigated both maximum reported side effects and the change in severity of side effects at multiple study timepoints in people with anxious and non-anxious depression over the course of treatment while controlling for multiple covariates. Given that we found no differences in either the maximum reported side effects or frequency, intensity, and burden of side effects across timepoints between people with anxious and non-anxious

Table 2. Correlations between QIDS-SR 16 Scores and FIBSER scores at each study timepoint.

\begin{tabular}{|c|c|c|c|c|c|c|c|c|c|c|c|c|c|}
\hline \multirow[t]{2}{*}{ Timepoint } & \multirow{2}{*}{$\begin{array}{l}\text { QIDS-SR }_{16} \\
M(\mathrm{SD})\end{array}$} & \multicolumn{4}{|c|}{ FIBSER-Frequency } & \multicolumn{4}{|c|}{ FIBSER-Intensity } & \multicolumn{4}{|c|}{ FIBSER-Burden } \\
\hline & & $M(\mathrm{SD})$ & $N$ & $r$ & $p$ & $M(\mathrm{SD})$ & $N$ & $r$ & $p$ & $M(\mathrm{SD})$ & $N$ & $r$ & $p$ \\
\hline Day 4 & $12.78(4.27)$ & $0.85(1.46)$ & 715 & 0.03 & 0.373 & $0.85(1.32)$ & 714 & 0.01 & 0.892 & $0.56(1.11)$ & 715 & 0.08 & 0.033 \\
\hline Week 4 & $9.20(4.72)$ & $1.34(1.56)$ & 673 & 0.24 & $<0.001$ & $1.40(1.38)$ & 673 & 0.27 & $<0.001$ & $0.90(1.20)$ & 673 & 0.33 & $<0.001$ \\
\hline Week 6 & $8.48(4.88)$ & $1.16(1.49)$ & 619 & 0.35 & $<0.001$ & $1.22(1.33)$ & 618 & 0.38 & $<0.001$ & $0.81(1.18)$ & 619 & 0.39 & $<0.001$ \\
\hline Week 8 & $7.63(4.72)$ & 1.19 (1.44) & 688 & 0.27 & $<0.001$ & $1.27(1.32)$ & 687 & 0.29 & $<0.001$ & $0.85(1.20)$ & 688 & 0.34 & $<0.001$ \\
\hline
\end{tabular}

FIBSER Frequency, Intensity, and Burden of Side Effects, QIDS-SR ${ }_{16}$ 16-item Quick Inventory of Depression Symptomatology-Self Reported. 


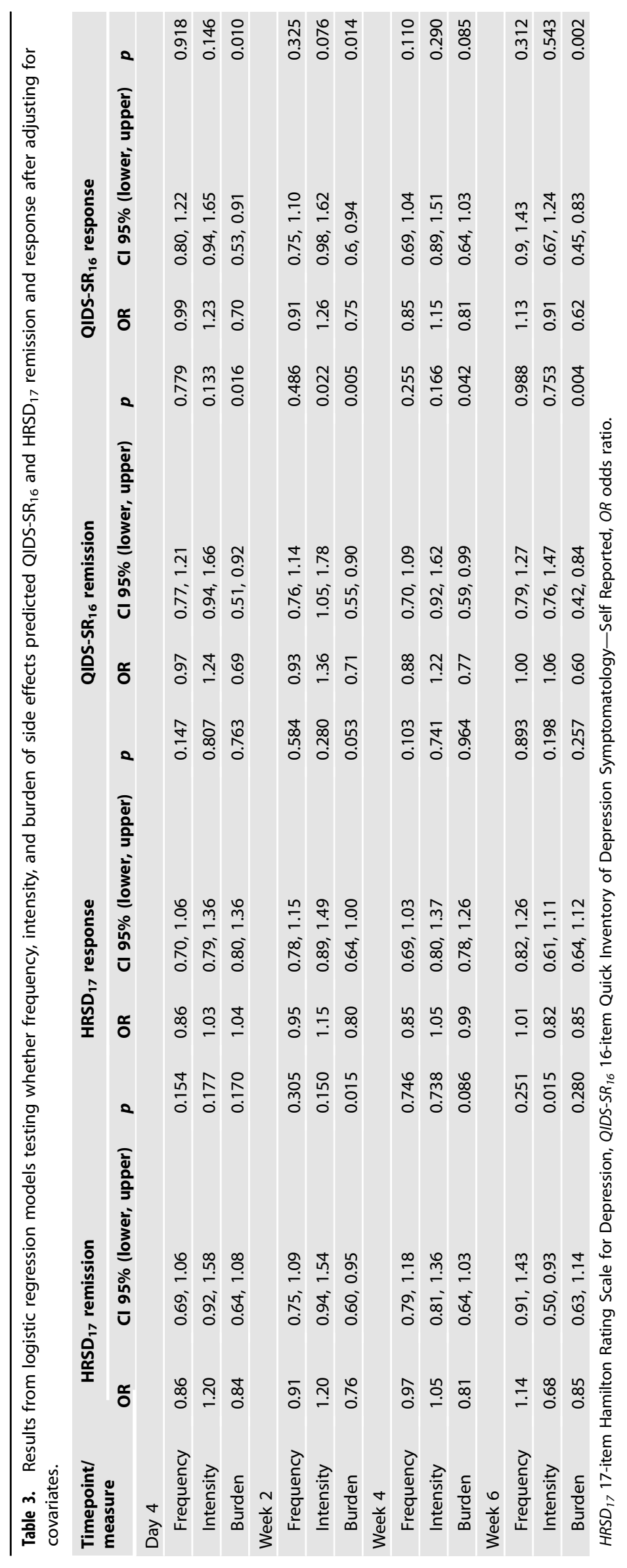


(A)
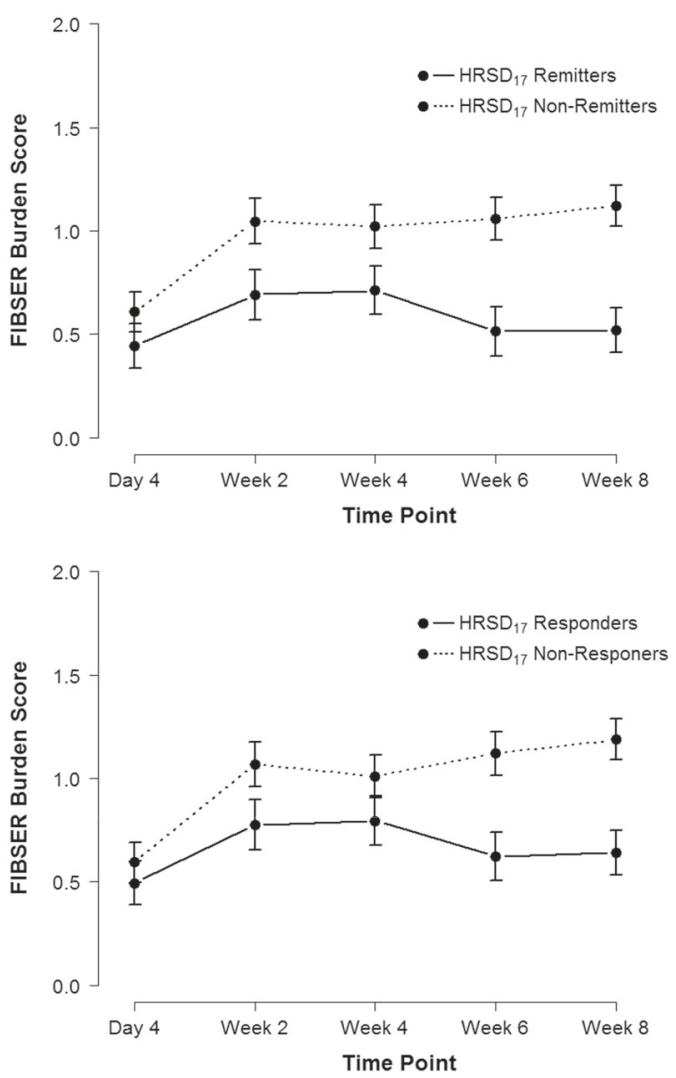

(B)
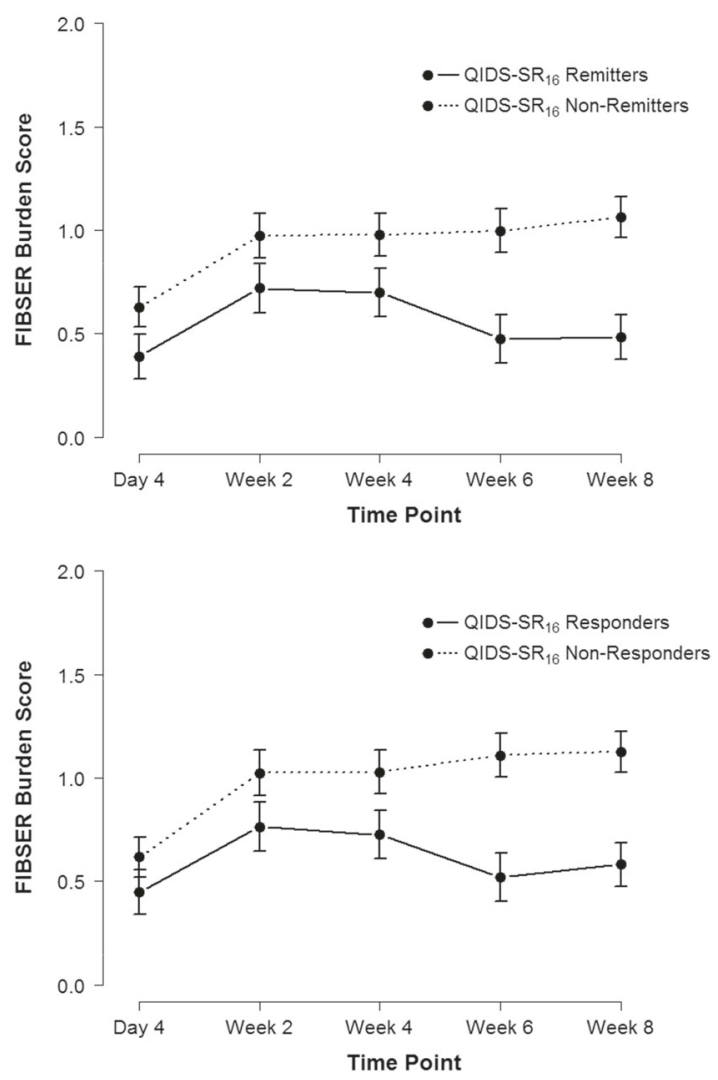

Fig. 2 Mean FIBSER burden score trajectories for remitters and responders. A HRSD 17 and B QIDS-SR 16 remitters and responders. Note: Error bars represent $95 \%$ confidence intervals of the mean.

depression, our difference in findings may be attributable to either a difference in the clinical severity of samples or lack of covariate controls. However, not all previous results are at odds with the current findings. For example, Gaspersz et al. [14]. found that syndromal anxious depression did not predict severity of side effects. Taken together, these results suggest that anxious symptom severity and the presence of anxious depression do not impact the severity of side effects over the course of antidepressant treatment.

A greater burden of side effects was associated with poorer QIDS-SR $_{16}$ remission and response at every timepoint, except for week 4 QIDS-SR 16 response. The association with poorer QIDS-SR $_{16}$ treatment outcome was present as early as 4 days post treatment. Moreover, the presence of adverse events were not associated with poorer treatment outcome. These results indicate that, in addition to side effects experienced throughout treatment, even initial antidepressant side effects can contribute towards poor treatment outcome. Moreover, the immediate impact of side effect burden is especially salient given depressive symptoms do not generally begin to resolve till weeks after initial treatment [37]. Associations between burden of side effects were only found for QIDS-SR 16 and not for the HRSD 17 , which may be related to the fact that both the QIDS-SR 16 and the FIBSER are self-reported, while the $\mathrm{HRSD}_{17}$ is clinician-rated. Furthermore, while the HRSD 17 is a multidimensional construct measuring both anxiety and depression, the QIDS-SR ${ }_{16}$ is a unidimensional construct measuring depression alone [38]. The $\mathrm{QIDS}^{-\mathrm{SR}_{16}}$ unilateral focus on depression may also align more closely with the FIBSER side effects related to antidepressant treatment compared to the HRSD $_{17}$. Nevertheless, greater burden of side effects presents as a prognostic marker of poorer QIDS-SR $_{16}$ treatment outcome and should be monitored closely.

This study has several limitations. First, our findings are limited to the antidepressants used in the current study, with other antidepressants requiring further investigation. Second, while side effect severity and general classes of side effects were assessed, specific side effects were not. Furthermore, side effects could not be matched with their corresponding severity. Therefore, the burden of some specific side effects may negatively impact treatment outcome more than others. While the FIBSER specifically targets side effects due to antidepressant medication, other protocol allowed non-psychotropic medications may have been misinterpreted for study treatment side effects and could impact the findings. Finally, because participants were diagnosed with DSM-IV criteria, the DSM-5 anxious distress specifier definition was not assessed [11].

Future research should aim to identify specific side effects that contribute to greater burden of side effect severity and poorer antidepressant treatment outcome. Given that burden of side effects not only predicted poorer treatment outcome but also failed to decrease throughout the course of treatment, a specific focus for future research should also be into the impacts of side effects causing enduring vs transient burden.

In conclusion, our results suggest that only frequency and intensity of side effects start to recede after at least 4 weeks of treatment and that anxious symptoms and the presence of anxious depression do not impact side effect severity. Furthermore, burden of side effects that are present as early as 4 days post-treatment predicts poorer treatment outcome and should be monitored closely. 


\section{REFERENCES}

1. Malhi GS, Mann JJ. Depression. Lancet. 2018;392:2299-312. https://doi.org/ 10.1016/S0140-6736(18)31948-2

2. Craske MG, Stein MB. Anxiety. Lancet. 2016;388:3048-59. https://doi.org/10.1016/ S0140-6736(16)30381-6

3. Wang SM, Han C, Bahk WM, Lee SJ, Patkar AA, Masand PS, et al. Addressing the side effects of contemporary antidepressant drugs: a comprehensive review. Chonnam Med J. 2018;54:101-12. https://doi.org/10.4068/cmj.2018.54.2.101

4. Moret $C$, Isaac M, Briley M. Problems associated with long-term treatment with selective serotonin reuptake inhibitors. J. Psychopharmacol. 2009;23:967-74. https://doi.org/10.1177/0269881108093582

5. Jakobsen JC, Katakam KK, Schou A, Hellmuth SG, Stallknecht SE, Leth-Møller K et al. Selective serotonin reuptake inhibitors versus placebo in patients with major depressive disorder. A systematic review with meta-analysis and tria sequential analysis. BMC Psychiatry. 2017;7:58. https://doi.org/10.1186/s12888016-1173-2

6. Saveanu R, Etkin A, Duchemin AM, Goldstein-Piekarski A, Gyurak A, Debattista C, et al. The international Study to Predict Optimized Treatment in Depression (iSPOT-D): outcomes from the acute phase of antidepressant treatment. J Psychiatr Res. 2015;61:1-12. https://doi.org/10.1016/j.jpsychires.2014.12.018

7. Trivedi $\mathrm{MH}$, Rush AJ, Wisniewski SR, Nierenberg AA, Warden D, Ritz L, et al. Evaluation of outcomes with citalopram for depression using measurementbased care in $\operatorname{STAR}^{*} \mathrm{D}$ : implications for clinical practice. Am J Psychiatry. 2006;163:28-40. https://doi.org/10.1176/appi.ajp.163.1.28

8. Ionescu DF, Niciu MJ, Richards EM, Zarate CA, Jr. Pharmacologic treatment of dimensional anxious depression: a review. Prim Care Companion CNS Disord 2014. https://doi.org/10.4088/PCC.13r01621.

9. Ionescu DF, Niciu MJ, Henter ID, Zarate CA. Defining anxious depression: a review of the literature. CNS Spectr. 2013;18:252-60. https://doi.org/10.1017/ S1092852913000114

10. Braund TA, Palmer DM, Williams LM, Harris AW. Characterising anxiety in major depressive disorder and its use in predicting antidepressant treatment outcome: an iSPOT-D report. Aust NZ J Psychiatry. 2019. https://doi.org/10.1177/ 0004867419835933

11. Gaspersz R, Nawijn L, Lamers F, Penninx B. Patients with anxious depression: overview of prevalence, pathophysiology and impact on course and treatment outcome. Curr Opin Psychiatry. 2018;31:17-25. https://doi.org/10.1097/ YCO.0000000000000376

12. Fava M, Rush AJ, Alpert JE, Balasubramani GK, Wisniewski SR, Carmin CN, et al. Difference in treatment outcome in outpatients with anxious versus nonanxious depression: a STAR*D report. Am J Psychiatry. 2008;165:342-51. https://doi.org/ 10.1176/appi.ajp.2007.06111868

13. Chan HN, Rush AJ, Nierenberg AA, Trivedi M, Wisniewski SR, Balasubramani GK, et al. Correlates and outcomes of depressed out-patients with greater and fewer anxious symptoms: a CO-MED report. Int J Neuropsychopharmacol. 2012;15:1387-99. https://doi.org/10.1017/S1461145711001660

14. Gaspersz R, Lamers F, Kent JM, Beekman A, Smit JH, van Hemert AM, et al. Anxious distress predicts subsequent treatment outcome and side effects in depressed patients starting antidepressant treatment. J Psychiatr Res. 2017;84:41-8. https://doi.org/10.1016/j.jpsychires.2016.09.018

15. Shankman SA, Gorka SM, Katz AC, Klein DN, Markowitz JC, Arnow BA, et al. Side effects to antidepressant treatment in patients with depression and comorbid panic disorder. J Clin Psychiatry. 2017;78:433-40. https://doi.org/10.4088/ JCP. $15 \mathrm{~m} 10370$

16. Williams LM, Rush AJ, Koslow SH, Wisniewski SR, Cooper NJ, Nemeroff CB, et al. International Study to Predict Optimized Treatment for Depression (iSPOT-D), a randomized clinical trial: rationale and protocol. Trials. 2011;12:4. https://doi.org/ 10.1186/1745-6215-12-4

17. Sheehan DV, Lecrubier $Y$, Sheehan $\mathrm{KH}$, Amorim $\mathrm{P}$, Janavs J, Weiller $\mathrm{E}_{\text {, et }}$ al. The Mini-International Neuropsychiatric Interview (M.I.N.I.): the development and validation of a structured diagnostic psychiatric interview for DSM-IV and ICD-10. J Clin Psychiatry. 1998;59:22-33.

18. American Psychiatric Association. Diagnostic and Statistical Manual of Mental Disorders. Vol. 4. Washington, DC: American Psychiatric Association; 1994.

19. Hamilton M. A rating scale for depression. J Neurol Neurosurg Psychiatry. 1960;23:56-62.

20. Rush AJ, Trivedi MH, Ibrahim HM, Carmody TJ, Arnow B, Klein DN, et al. The 16Item Quick Inventory of Depressive Symptomatology (QIDS), clinician rating (QIDS-C), and self-report (QIDS-SR): a psychometric evaluation in patients with chronic major depression. Biol Psychiatry. 2003;54:573-83. https://doi.org/ 10.1016/s0006-3223(02)01866-8

21. Trivedi $M H$, Rush AJ, Ibrahim HM, Carmody TJ, Biggs MM, Suppes $T$, et al. The Inventory of Depressive Symptomatology, Clinician Rating (IDS-C) and Self-Report (IDS-SR), and the Quick Inventory of Depressive Symptomatology, Clinician Rating (QIDS-C) and Self-Report (QIDS-SR) in public sector patients with mood disorders: a psychometric evaluation. Psychol Med. 2004;34:73-82. https://doi. org/10.1017/s0033291703001107

22. Trajković $G$, Starčević $V$, Latas $M$, Leštarević $M$, Ille T, Bukumirić $Z$, et al. Reliability of the Hamilton Rating Scale for Depression: a meta-analysis over a period of 49 years. Psychiatry Res. 2011;189:1-9. https://doi.org/10.1016/j.psychres.2010.12.007

23. Cameron IM, Crawford JR, Cardy AH, du Toit SW, Lawton K, Hay S, et al. Psychometric properties of the Quick Inventory of Depressive Symptomatology (QIDS-SR) in UK primary care. J Psychiatr Res. 2013;47:592-8. https://doi.org/ 10.1016/j.jpsychires.2013.01.019

24. Wisniewski SR, Rush AJ, Balasubramani GK, Trivedi MH, Nierenberg AA. Self-rated global measure of the frequency, intensity, and burden of side effects. J Psychiatr Pract.2006;12:71-9.

25. Brown EG, Wood L, Wood S. The medical dictionary for regulatory activities (MedDRA). Drug Saf. 1999;20:109-17. https://doi.org/10.2165/00002018199920020-00002

26. Cleary P, Guy W. Factor analysis of the Hamilton Depression Scale. Drugs Exp Clin Res. 1977;1:115-20.

27. Lovibond PF. Long-term stability of depression, anxiety, and stress syndromes. J. Abnorm Psychol. 1998;107:520-6.

28. Lovibond SH, Lovibond PF. Manual for the Depression Anxiety Stress Scales. 2nd ed. Sydney, NSW: Psychology Foundation; 1995.

29. Akaike H. A new look at the statistical model identification. In: Parzen $\mathrm{E}$, Tanabe $\mathrm{K}$ Kitagawa G, editors. Selected papers of Hirotugu Akaike. New York, NY: Springer 1974. p. 215-22.

30. Schwarz G. Estimating the dimension of a model. Ann Stat. 1978;6:461-4.

31. Holm S. A simple sequentially rejective multiple test procedure. Scand J Stat. 1979;6:65-70.

32. R Core Team. R: A language and environment for statistical computing. Vienna: $R$ Foundation for Statistical Computing; 2020.

33. Bates D, Machler M, Bolker BM, Walker SC. Fitting linear mixed-effects models using Ime4. J Stat Softw. 2015;67:1-48.

34. Kuznetsova A, Brockhoff PB, Christensen RHB. ImerTest Package: tests in linear mixed effects models. J Stat Softw. 2017:82:1-26.

35. Lenth R. mmeans: Estimated marginal means, aka least-squares means. 2019 Retrieved from https://CRAN.R-project.org/package=emmeans.

36. Sansone RA, Sansone LA. Antidepressant adherence: are patients taking their medications? Innov Clin Neurosci. 2012;9:41-6.

37. Stahl SM. Stahl's essential psychopharmacology: neuroscientific basis and practical application. 4th ed. Cambridge: Cambridge University Press; 2013.

38. McClintock SM, Haley C, Bernstein IH. Psychometric considerations of depression symptom rating scales. Neuropsychiatry. 2011;1:611-23. https://doi.org/10.2217/ Npy.11.59

\section{ACKNOWLEDGEMENTS}

We thank all the patients and volunteers who agreed to participate in these studies. We acknowledge Brain Resource as the sponsor for the iSPOT-D study (NCT00693849). We acknowledge the roles of Evian Gordon, Ph.D. MD, as sponsor Principal Investigator, Leanne Williams, Ph.D., as the cross-site academic Principal Investigator for iSPOT-D (2008-2013), and Claire Day, Ph.D., as the global trial coordinator for iSPOT-D (2008-2014), and the iSPOT-D Publication Team. TAB was supported by a NSW Health PhD scholarship. iSPOT-D was sponsored by Brain Resource Company Operations Pty Ltd. Brain Resource personnel coordinated the research sites and data quality control but did not participate in the collection of any data.

\section{AUTHOR CONTRIBUTIONS}

TAB: conceptualization, formal analysis, writing-original draft, writing-review and editing. GT, DMP, EG, AJR, AWFH: conceptualization, writing-review and editing.

\section{COMPETING INTERESTS}

$T A B, D M P$, and EG report salaries from Total Brain outside the submitted work. EG is the $\mathrm{CMO}$ at Total Brain and holds significant equity and stock options in the company. AWFH reports personal fees from Janssen Australia, Lundbeck Australia, Servier Australia, and Takeda Pharmaceutical Company outside the submitted work. AJR has received consulting fees from Akili, Brain Resource Inc., Compass Inc., Curbstone Consultant LLC, Emmes Corp., Holmusk, Inc., Liva-Nova, Johnson \& Johnson (Janssen), Sunovion, Takeda USA, and Taj Medical; speaking fees from LivaNova; and royalties from Guilford Press and the University of Texas Southwestern Medical Center, Dallas, TX (for the Inventory of Depressive Symptoms and its derivatives). He is also named co-inventor on two patents: US Patent No. 7,795,033: Methods to predict the outcome of treatment with antidepressant medication, Inventors: McMahon FJ, Laje G, Manji H, Rush AJ, Paddock S, Wilson AS and US Patent No. 7,906,283: Methods to identify patients at risk of 


\section{8}

developing adverse events during treatment with antidepressant medication, Inventors: McMahon FJ, Laje G, Manji H, Rush AJ, Paddock S. GT has no conflicts of interest to report.

\section{ADDITIONAL INFORMATION}

Supplementary information The online version contains supplementary material available at https://doi.org/10.1038/s41398-021-01533-1.

Correspondence and requests for materials should be addressed to T.A.B.

Reprints and permission information is available at http://www.nature.com/reprints

Publisher's note Springer Nature remains neutral with regard to jurisdictional claims in published maps and institutional affiliations. (i) Open Access This article is licensed under a Creative Commons Attribution 4.0 International License, which permits use, sharing, adaptation, distribution and reproduction in any medium or format, as long as you give appropriate credit to the original author(s) and the source, provide a link to the Creative Commons license, and indicate if changes were made. The images or other third party material in this article are included in the article's Creative Commons license, unless indicated otherwise in a credit line to the material. If material is not included in the article's Creative Commons license and your intended use is not permitted by statutory regulation or exceeds the permitted use, you will need to obtain permission directly from the copyright holder. To view a copy of this license, visit http://creativecommons. org/licenses/by/4.0/.

(c) The Author(s) 2021 